\title{
Sepsis Produces Microthrombosis and Not Coagulation: Implications for Sepsis Therapy
}

\author{
H Bradford Hawley, MD* \\ Department of Internal Medicine Boonshoft School of Medicine, Wright State University, Dayton, Ohio, USA
}

\begin{abstract}
Summary
A new theory of hemostasis proposes microthrombosis, not coagulation, as a key event in sepsis. Endotheliopathy releases unusually large von Willebrand factor which complexes with platelets to form organ damaging microthrombi. This theory holds the promise for more effective sepsis therapy.
\end{abstract}

\begin{abstract}
The hemostatic abnormalities which may accompany sepsis have been attributed to disseminated intravascular coagulation (DIC) initiated by tissue factor (TF) activating FVII leading to TF-FVII a complex formation and triggering the extrinsic coagulation cascade. Recently, a new theory of hemostasis has been proposed in which sepsis is associated with endothelial damage releasing unusually large von Willebrand factor (ULVWF). The ULVWF multimers, following exocytosis from sepsis-induced endothelialopathy, become anchored to the endothelial cell membrane as elongated strings which recruit platelets from the circulation forming platelet-ULVWF complexes which become microthrombi. The process is called microthrombogenesis and the resultant disorder is disseminated intravascular thrombosis (DIT) which is a major factor leading to organ dysfunction. Thus, the sepsis-associated coagulopathy is not a coagulation disorder, but is a microthrombotic disorder. This conceptual reinterpretation entails a significant paradigm shift in the understanding of sepsis pathophysiology and treatment.
\end{abstract}

\section{Keywords}

Sepsis, Endothelium, Von Willebrand Factor, Microthrombosis

\section{Introduction}

Sepsis is a condition, not a disease, and as such demands a broader understanding of pathogenesis and treatment. In 2014, a third international consensus conference was convened to re-examine the definition of sepsis. They defined sepsis as "life-threatening organ dysfunction caused by a dysregulated host response to infection" [1]. This definition recognized the importance of organ dysfunction to the septic condition and outcome. The organ failure in septic patients is often the result of disseminated microthrombi within the micro and small vasculature [2-4]. These microthrombi have been thought to be the result of a coagulation disorder currently termed disseminated intravascular coagulation (DIC). This process has been attributed to tissue factor (TF) mediated thrombin generation and formation of fibrin clots (extrinsic pathway) $[3,4]$. Recently the pathogenesis of DIC has been redefined based upon not only the characteristic data of coagulation and the failure of traditional anticoagulation therapy in clinical trials, but also on a new theory of in vivo hemostasis. This theory clarifies that the nature of microthrombi in sepsis is not fibrin clots (Figure 1) [5].

\section{Extrinsic Pathway and Tissue Factor Release}

The extrinsic pathway is so named because it requires TF which is located in vascular smooth muscle cells, pericytes, and adventitial fibroblasts "extrinsic" to the endothelium of blood vessels to initiate coagulation. Injury to the vascular wall can occur in several ways. Traumatic injury, vascular diseases, and surgery (including vascular procedures) can all cause both endothelial cell damage as well as damage to subendothelial and extravascular tissue. In these types of injuries TF, a transmembrane receptor on cells in the subendothelial and intravascular tissue, can come into contact with circulat-

*Corresponding author: Dr. H Bradford Hawley, MD, Department of Internal Medicine Boonshoft School of Medicine, Wright State University, 6761 Trailview Drive, Dayton, $\mathrm{OH}$ 45414, USA, Tel: 937-898-2153

Accepted: November 10, 2020

Published online: November 12, 2020

Citation: Hawley HB (2020) Sepsis Produces Microthrombosis and Not Coagulation: Implications for Sepsis Therapy. Ann Public Health Reports 4(1):87-91 
ing coagulation factors. The combination of TF with activated coagulation factor VII initiates the coagulation cascade [6]. This pathway has been referred to as the extrinsic pathway. Other investigators have proposed that during sepsis cytokine stimulation will cause endothelial cells and circulating blood cells, such as monocytes, to express TF $[7,8]$. The expression of TF by endothelial cells and circulating monocytes has only been demonstrated in cell culture studies which expose cells to some harsh conditions making their validity questionable [9]. In vivo studies in animals and humans have measured small amounts of circulating TF under septic conditions, but all these studies have been performed when the vascular wall and endothelium have been punctured by intravascular catheters and multiple blood drawing needles. Indeed, "direct evidence is scarce in humans" with respect to demonstrating TF on endothelial cells during sepsis [10].

\section{New Theory of Hemostasis}

According to the new theory of in vivo hemostasis, following vascular injury, a microthrombogenesis path becomes ac- tivated due to endothelial damage and a fibrinogenesis path is activated due to subendothelial damage [11]. When the vascular injury involves both the endothelium and the subendothelial tissue, both the microthrombogenic and fibrinogenic pathways become active. The products of these pathways can co-mingle and passively trap blood cells to finally result in a blood clot (macrothombogenesis). These 3 processes (microthrombogenesis, fibrinogenesis, and macrothrombogenesis) and their pathways are simply illustrated in (Figure 1).

TF is released when injury extends beyond the subendothelial and extravascular tissue and leads to fibrinogenesis. In sepsis, the complement system affords important protection against some invading pathogens such Neisseria meningitidis. Activation of the complement system occurs via the alternative, classical (antibody requiring), or lectin pathways and while the initial steps vary for each pathways they all lead to the formation of C3. C3 is cleaved by C3 converts to form $\mathrm{C} 3 \mathrm{~b}$ which is able to bind to the surfaces of both pathogens and host cells. The process continues with the cleavage of

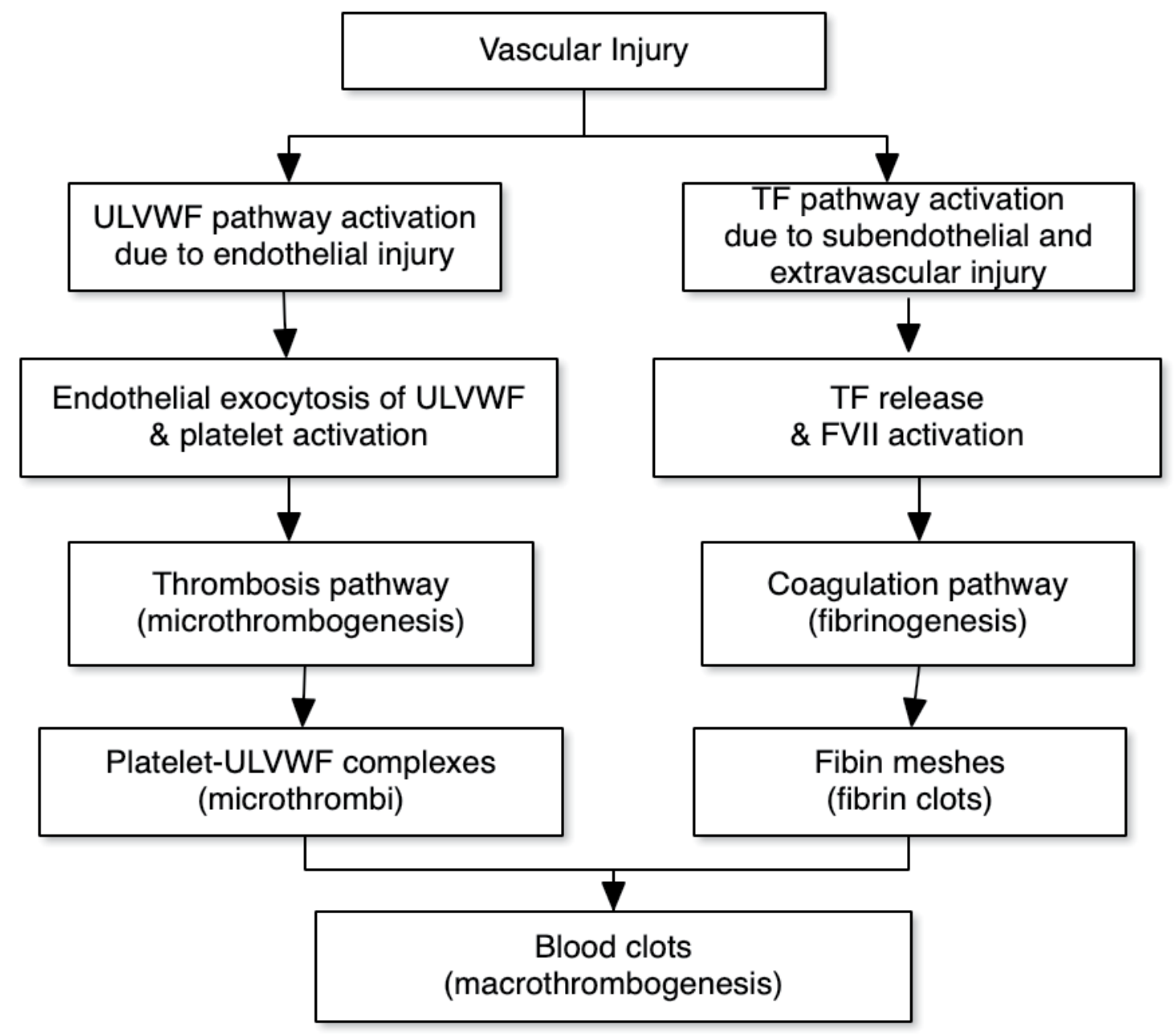

Figure 1: New Theory of Hemostasis.

A new theory of hemostasis has been derived from the physiological logic of in vivo hemostasis based on the level (depth) of vascular injury. Sepsis is characterized by endothelial injury without subendothelial and extravascular injury. This intravascular process initiated by endothelial injury results in release of unusually large von Willebrand factor (ULVWF) which traps platelets. The platelet-ULVWF complexes form microthrombi. Co-mingling of the platelet-ULVWF complexes of the thrombosis pathway with the fibrin meshes of the coagulation pathway produce blood clots (macrothrombi). 
Citation: Hawley HB (2020) Sepsis Produces Microthrombosis and Not Coagulation: Implications for Sepsis Therapy. Ann Public Health Reports 4(1):87-91

C5 into $\mathrm{C} 5 \mathrm{a}$ and $\mathrm{C} 5 \mathrm{~b}$ by $\mathrm{C} 5$ convertase. $\mathrm{C} 5 \mathrm{~b}$ then initiates the assembly of the final active component of the complement system termed the membrane attack complex (MAC). MAC lyses pathogens, such as $N$. meningitidis by causing pores in cell membranes, but also has the potential to cause damage to host cells if not protected [12,13]. The formation of MAC is inhibited by a membrane protein called CD59 which is present in normal host cells but not in microbes. CD59 is found in the membranes of blood cells, endothelial cells, and epithelial cells and blocks the terminal addition of C9 which finally completes the active membrane attack complex. MAC is inhibited to a lesser extent by two other membrane proteins (DAF/CD55 and MCP/CD46) as well as protein S which circulates in the serum. Endotheliopathy may also be caused by pathogens directly or by binding to endothelial receptors. The binding of SARS-CoV-2 to ACE2 receptors on endothelial cells is an example of this direct endotheliopathy. The resulting endotheliopathy activates platelets and causes the exocytosis of unusually large von Willebrand factor multimers (ULVWF) from Weibel-Palade bodies within the endothelial cells. The ULVWF becomes anchored to membranes of the damaged endothelial cells forming long strings that can recruit and bind platelets. Because of shear stress the strings always dangle from the surface of endothelial cells in the same direction as the blood flow. These complexes become microthrombi composed of platelets and ULVWF $[11,14]$. ADAMTS13 is a protease that cleaves ULVWF limiting microthrombogenesis. During sepsis levels of ADAMTS13 in patients are decreased compared with patients having non-infectious systemic inflammatory response syndrome [15]. The reasons for this are as yet undetermined, but may be due to consumption by the large amount of ULVWF generated at endothelial surfaces during sepsis [16]. This pathological process has been termed endotheliopathy-associated vascular microthrombotic disease (EA-VMTD) [11].

\section{Disseminated Intravascular Coagulation and Thrombosis}

Despite the difficulties in finding the exact source of tissue factor, it has continued to be regarded as the trigger for the hemostatic disorder called DIC [4]. DIC has been associated with a variety of disease states in addition to sepsis. The new theory of hemostasis proposes that "true" DIC only occurs in acute promyelocytic leukemia (APL) [16]. In APL the abnormal leukemic cells express TF, producing true DIC through an alternate pathway $[17,18]$. Since true DIC is a consumptive coagulopathy, the levels of FVIII in the blood of patients should be decreased and they are. However, in other types of DIC levels of FVIII in the blood of patients are increased, a fact that is inconsistent with consumptive coagulation and a fibrinogenesis pathway [16]. In a series of 26 patients from the Massachusetts General Hospital, published in 1972 to present the pathology of DIC, the underlying disease in 3 of the patients was APL [2]. This is remarkable in that APL is a relatively rare disease that occurs in about 1 out of every 250,000 individuals in the population. The EA-VMTD seen in septic patients is a microthrombotic disorder whereas true DIC, as accompanies APL, is a coagulation disorder resulting in fibrin clots. The correct terminology for the microthrombotic dis- order resulting from EA-VMTD is disseminated intravascular microthrombosis (DIT) [15]. Thus DIC is a hemostatic coagulation disorder and DIT is a microthrombotic disorder.

\section{Complement Disorders and Thrombosis}

Deficiencies of complement components greatly increase the risk of infection by Neisseria meningitidis. Meningococcal infection occurs in the majority of these individuals as a result of the failure to form MAC which is able to kill vulnerable meningococcal. Patients not only have first episodes but suffer relapses, recurrences, and multiple episodes. Despite their increased susceptibility to meningococcal infection, the disease is much milder with a higher survival rate than observed in patients without complement deficiencies. The reasons for this are unclear, but less severe DIT may play a role.

A genetic defect resulting in the inability of the complement regulating protein $\mathrm{CD} 59$ to anchor to the cell membrane causes paroxysmal nocturnal hemoglobinuria. In addition to the complement mediated hemolysis these patients are at increased risk for thrombosis. The thrombosis risk occurs because of the unregulated MAC producing endotheliopathy with release of ULVWF. This disease is treated with the anti-complement monoclonal antibody eculizumab.

\section{Sepsis Causes Multiple Organ Dysfunction}

Critical care physicians now recognize that organ dysfunction is the major determinant of sepsis mortality [19]. Since EA-VMTD/DIT plays a major role in life-threatening organ dysfunction, it is critical to understand the structure and function of the endothelium in various organs. In fact, endothelial cell phenotypes differ not only between different organ types but also within the vascular segments of the same organ [7]. These differences are both structural and functional and are determined by both genetics and the extracellular milieu. When epithelial cells are grown in tissue culture apart from their usual extracellular environment they undergo phenotypic drift. Some genes are stable, but as many as 50\% may lose or alter their function in tissue culture. Such changes may explain some of the studies demonstrating tissue factor expression by endothelial cells in tissue culture [20]. In addition to the heterogeneity of endothelial cells, the invading pathogen may exhibit host organ specificity [2]. In septic patients microthrombi can be found in many organs. Autopsies of septic patients have revealed the organ involvement is variable [7]. Kidney and lung microthrombi were the most common with each being found in $70 \%$ of patients. Brain, heart, liver, spleen, and adrenals were each involved in about $40 \%$ of patients. Pancreas and gut thrombi were the least common and were seen in only about $20 \%$ of patients. Of course, these autopsy figures represent septic patients in general with sepsis resulting from a wide variety of pathogens. If organ involvement with thrombi were examined in patients with a unique pathogen etiology, the organ involvement would likely be different and characteristic of that pathogen [21]. An example of pathogen specific organ involvement would be the adrenal tropism of Neisseria meningitidis causing sepsis and Waterhouse-Friderichsen syndrome. The distribution of macrothrombi found at autopsy corresponds to the failing organs 
suggesting a causal relationship. Multiple organ dysfunction and failure frequently accompany severe sepsis and a greater reduction in microvascular blood flow is seen in nonsurvivors [22].

\section{Thrombocytopenia and Sepsis}

A study of septic patients admitted to the intensive care unit demonstrated that nearly half of the patients had thrombocytopenia [23]. Thrombocytopenic patients who did not resolve their thrombocytopenia had an increased mortality. Platelets are consumed by the formation of microthrombi in blood vessels [24]. The DIT causes not only thrombocytopenia but also microangiopathic hemolytic anemia [25]. The combination of unexplained thrombocytopenia and hemolytic anemia may be a sign of sepsis and should prompt immediate investigation. The microthrombi that are anchored to blood vessels of all sizes create a thrombotic thrombocytopenia purpura (TTP)-like syndrome.

\section{Sepsis Therapies: Past and Future}

The definitive treatment of sepsis requires control of the infection and elimination of the invading microorganism. While the results of various diagnostic modalities are pending, empiric antimicrobial therapy is recommended [26]. The recipe for successful empiric antibiotics may be still debated but the wisdom of early, effective therapy is not [27]. Inappropriate antimicrobial therapy decreases survival [28]. The most aggressive approach requires the septic patient to be seen by an infectious disease consultant within one hour after arrival in the emergency department [29].

Pathogen elimination requires time and during that interval measures to support the patient are required. Early goal-directed therapy centered on intravenous fluids and pharmacological measures to maintain the circulation appeared to have great benefit only to be later shown to be less beneficial [30,31]. Vitamin C therapy remains controversial with as yet unproven mechanisms of action as well as benefit [32]. A variety of agents, including antithrombin III, recombinant activated protein C (drotrecogin alpha), and heparin, aimed at interrupting the coagulation cascade have all proven ineffective in the treatment of sepsis [33]. The failure of these therapies directed at the extrinsic pathway provides evidence that it is not this pathway that is responsible for the hemostatic abnormalities.

Microthrombogenesis caused by endotheliopathy and release of ULVWF must be ameliorated if organ dysfunction is to be reduced and survival rate improved for septic patients. The theoretically ideal candidate to investigate as a therapeutic agent is ADAMTS13. A recombinant form of ADAMTS13 has been used successfully to treat the early stages of TTP in rats [34]. Recombinant ADAMTS13 has also been employed to restore the von Willebrand factor cleaving activity of ADAMTS13 in plasma obtained from patients with acquired TTP [35]. Since EA-VMTD/DIT of sepsis is a TTP-like illness, a similar approach using recombinant ADAMTS13 might be a fruitful avenue of research in animals and humans with sepsis.
Recently studies of Staphylococcus aureus sepsis have found that the ratio of VWF/ADAM13TS correlated with disease severity and outcome in both human patients and mice [36]. Immunotherapy with caplacizumab has recently been approved in both Europe and the United States for the treatment of acquired TTP [37]. This antibody targets von Willebrand factor and might also be of benefit for sepsis therapy. Pulmonary microscopic findings from autopsies of patients succumbing from COVID-19 reveal "fibrin thrombi present within distended small vessels and capillaries" with von Willebrand factor being visible in these vessels with immunostaining [38]. Eculizumab is a long acting monoclonal antibody against complement $\mathrm{C} 5$ which is used to treat paroxysmal nocturnal hemoglobinuria. It prevents cleavage of C5 into $\mathrm{C} 5 \mathrm{a}$ and $\mathrm{C} 5 \mathrm{~b}$ inhibiting formation of MAC which can produce DIT as previously described. A clinical trial of eculizumab for the treatment of COVID-19 is currently underway (SOLID-C19). These are just a few of the examples of research efforts with potential benefit that can be pursued with recognition that thrombosis (EA-VMTD/DIT), not coagulation (DIC), is responsible for the hemostatic disorder accompanying sepsis and leading to organ dysfunction and increased mortality.

\section{Acknowledgement}

The author would like to thank Dr. Jae Chang for his encouragement and critical review of the manuscript.

\section{Funding}

None.

\section{Conflicts of Interest}

None.

\section{Authorship}

The author is solely responsible for the content of this manuscript.

\section{References}

1. Singer M, Deutschman CS, Seymour CW, et al. (2016) The third international consensus definitions for sepsis and septic shock (Sepsis-3). JAMA 315: 801-810.

2. Robboy SJ, Major MC, Colman RW, et al. (1972) Pathology of disseminated intravascular coagulation: (DIC). Hum Pathol 3: 327-343.

3. Levi M, Cate HT (1999) Disseminated intravascular coagulation. N Engl J Med 341: 586-592.

4. Papageorgiou C, Jourdi G, Adjambri E, et al. (2018) Disseminated intravascular coagulation: an update on pathogenesis, diagnosis, and therapeutic strategies. Clin Appl Thomb Hemost 24: 8S-28S.

5. Chang JC (2019) Acute respiratory distress syndrome as an organ phenotype of vascular microthrombotic disease: Based on hemostatic theory and endothelial molecular pathogenesis. Clin Appl Thromb Hemost 25: 1-20.

6. Mackman N (2009) The role of tissue factor and factor VIla in hemostasis. Anesth Analg 108: 1447-1452.

7. Levi M, van der Poll T, Schultz M (2012) Systemic versus localized coagulation activation contributing to organ failure in critically ill patients. Semin Immunpathol 34: 167-179. 
8. Levi M, van der Poll, T (2010) Inflammation and coagulation. Crit Care Med 38: S26-S34.

9. Osterud B, Bjorklid E (2006) Sources of tissue factor. Semin Thromb Hemost 32: 11-23.

10. Ait-Oufella H, Maury E, Leboux S, et al. (2010) The endothelium: Physiological functions and role in microcirculatory failure during sepsis. Intensive Care Med 36: 1286-1298.

11. Chang JC (2018) Hemostasis based on a novel 'two-path unifying theory' and classification of hemostatic disorders. Blood Coag Fibrinol 29: 573-584.

12. Kerr H, Richards A (2012) Complement-mediated injury and protection of endothelium: lessons from the hemolytic uremic syndrome. Immunobiology 217: 195-203.

13. Helling H, Stephan B, Pindur G (2015) Coagulation and complement system in critically ill patients. Clin Hemorheol Microcirc 61: 185-193.

14. Chang JC (2017) Thrombocytopenia in critically ill patients due to vascular microthrombotic disease: Pathogenesis based on "two-activation theory of the endothelium." Vasc Dis Ther 2: 1-7.

15. Albar J, Castro P, Espinosa G, et al. (2015) ADAMTS-13 in critically ill patients with septic syndromes and noninfectious systemic inflammatory response syndrome. Shock 43: 556-562.

16. Chang JC (2018) Disseminated intravascular coagulation: is it fact or fancy? Blood Coag Fibrinol 29: 330-337.

17. Cooperberg AA (1967) Acute promyelocytic leukemia. Can Med Assoc J 97: 57-63.

18. Dunoyer-Geindre S, Rivier-Cordey AS, Tsopra O, et al. (2017) Effect of ATRA and ATO on the expression of tissue factor in NB4 acute promyelocytic leukemia cells and regulatory function of the inflammatory cytokines TNF and IL-1B. Ann Hematol 96: 905917.

19. Jacob JA (2016) New sepsis diagnostic guidelines shift focus to organ dysfunction. JAMA 315: 739-740.

20. Szotowski B, Antoniak S, Poller W, et al. (2005) Procoagulant soluble tissue factor is released from endothelial cells in response to inflammatory cytokines. Circ Res 96: 1233-1239.

21. Chang JC (2019) Sepsis and septic shock endothelial molecular pathogenesis associated with vascular microthrombotic disease. Thromb J 17: 10.

22. Vincent JL, De Becker D (2005) Microvascular dysfunction as a cause of organ dysfunction in severe sepsis. Crit Care 9: S9-S12.

23. Venkata C, Kashyap R, Farmer R, et al. (2013) Thrombocytopenia in adult patients with sepsis: Incidence, risk factors, and its association with clinical outcome. J Intensive Care 1: 9.

24. Greco E, Lupia E, Bosco O, et al. (2017) Platelets and multi-organ failure in sepsis. Int J Mol Sci 18: 2200.
25. Chang JC (2018) TTP-like syndrome: Novel concept and molecular pathogenesis of endotheliopathy-associated vascular microthrombotic disease. Thrombosis Journal 16: 20.

26. Septimus EJ, Coopersmith CM, Whittle J, et al. (2017) Sepsis national hospital quality measure (SEP-1): Multistakeholder work group recommendations for appropriate antibiotics for the treatment of sepsis. Clin Infect Dis 65: 1565-1569.

27. IDSA Sepsis Task Force (2018) Infectious Diseases Society of America (IDSA) POSITION STATEMENT: Why IDSA did not endorse the surviving sepsis campaign guidelines. Clin Infect Dis 66: 1631-1635.

28. Kumar A, Ellis P, Arabi Y, et al. (2009) Initiation of inappropriate antimicrobial therapy results in a fivefold reduction of survival in human septic shock. Chest 136: 1237-1248.

29. Viale P, Tedeschi S, Scudeller L, et al. (2017) Infectious diseases team for the early management of severe sepsis and septic shock in the emergency department. Clin Infect Dis 65: 12531259.

30. Rivers E, Nguyen B, Havstad S, et al. (2001) Early goal-directed therapy collaborative group: early goal-directed therapy in the treatment of severe sepsis and septic shock. N Engl J Med 345: 1368-1375.

31. The PRISM Investigators, Kathryn M Rowan, Derek C Angus, et al. (2017) Early goal-directed therapy for septic shock-a patient level meta-analysis. N Engl J Med 376: 2223-2234.

32. Brant EB, Angus DC (2019) Is high-dose vitamin C beneficial for patients with sepsis? JAMA 322: 1257-1258.

33. Crowther MA, Marshall JC (2001) Continuing challenges of sepsis research. JAMA 286: 1894-1896.

34. Tersteeg C, Schiviz A, De Meyer SF, et al. (2015) Potential for recombinant ADAMTS13 as an effective therapy for acquired thrombotic thrombocytopenia purpura. Arterioscler Thromb Vasc Biol 35: 2336-2342.

35. Plaimauer B, Kremer Hovinga JA, Juno C, et al. (2011) Recombinant ADAMTS13 normalizes von Willebrand factor-cleaving activity in plasma of acquired TTP patients by overriding inhibitory antibodies. J Thromb Haemostas 9: 936-944.

36. Peetermans M, Meyers S, Liesenborghs L, et al. (2020) Von Willebrand factor and ADAMTS13 impact on the outcome of Staphylococcus aureus sepsis. J Thromb Haemost 18: 722-731.

37. Scully M, Cataland SR, Peyvandi P, et al. (2019) Caplacizumab treatment for acquired thrombocytopenic purpura. N Engl J Med 380: 335-346.

38. Sharon E Fox, Aibek Akmatbekov, Jack L Harber, et al. (2020) Pulmonary and cardiac pathology in Covid-19: The first autopsy series from New Orleans. med Rxiv BMJ.

DOI: $10.36959 / 856 / 501$

Copyright: (C) 2020 Hawley HB. This is an open-access article distributed under the terms of the Creative Commons Attribution License, which permits unrestricted use, distribution, and reproduction in any medium, provided the original author and source are credited. 